\title{
The Anatomy of Ultrahigh-Energy Cosmic Rays
}

\author{
New results from the Pierre Auger Observatory could narrow down the \\ search for the origin of ultrahigh-energy cosmic rays.
}

By Soebur Razzaque

I Itrahigh-energy cosmic rays (UHECRs) at energies $10^{18} \mathrm{eV}$ and above are the most energetic subatomic particles in nature. To get an idea of their energy, the most energetic of these particles carries the same punch $\left(10^{20} \mathrm{eV}\right)$ as a tennis ball coming off a racket with a speed of 100 $\mathrm{km} / \mathrm{h}$. For comparison, the Large Hadron Collider (LHC) at CERN can accelerate protons to only about $10^{13} \mathrm{eV}$. The tremendous energy of UHECRs naturally begs questions of how and where they are produced. Although discovered almost 60 years ago [1], the origin and chemical composition of UHECRs are still

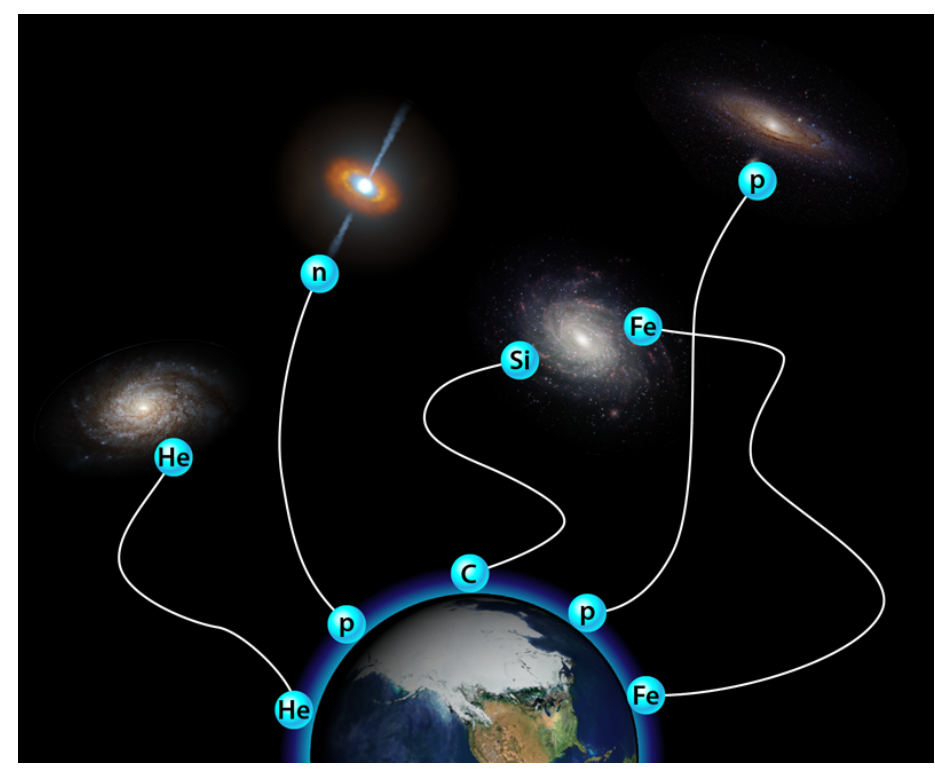

Figure 1: The latest data from the Pierre Auger Observatory suggest that ultrahigh-energy cosmic rays are a mix of nuclei that arrive from a large collection of galaxies spread evenly over the sky. Credit: APS/Alan Stonebraker (galaxy images from NASA) unknown. The latest data from the Pierre Auger

Observatory-collected over more than a decade-provide the largest sample to date of UHECRs, with over 215,000 events [2]. The observations reveal a new feature-a steepening in the spectrum that joins other spectral features like the "knee" and the "ankle." The data also show that UHECRs arrive uniformly over the sky. Together, these results suggest that energetic star factories, called starburst galaxies, might be the most promising sources for UHECRs.

The Pierre Auger Observatory [3] in Argentina and the Telescope Array (TA) [4] in the US are the two largest cosmic-ray detectors currently operational, covering 3000 and 700 square kilometers of instrumented areas, respectively. Such huge areas are required to detect UHECRs, which reach Earth with a flux of only about a hundred per square kilometer per year (Fig. 1). At ultrahigh energies, cosmic rays break up in the atmosphere by interacting with the air molecules, creating approximately a billion or more secondary particles, which shower down on Earth's surface. Such an event is aptly called an extended air shower (EAS). An array of surface detectors can reconstruct the energy and direction of the primary UHECR by detecting the EAS particles (mostly muons) that reach the ground. The accuracy of measurements increases significantly by using telescopes at night to observe fluorescence light from the EAS particles exciting the nitrogen molecules in the air. Both the Auger and TA employ this hybrid technique to detect UHECRs.

One of the most hotly debated issues in the cosmic-ray community is the chemical composition of UHECRs. At lower energies, satellite and balloon-borne experiments directly measure the primary cosmic rays, allowing them to determine the composition as predominantly protons and nuclei with 


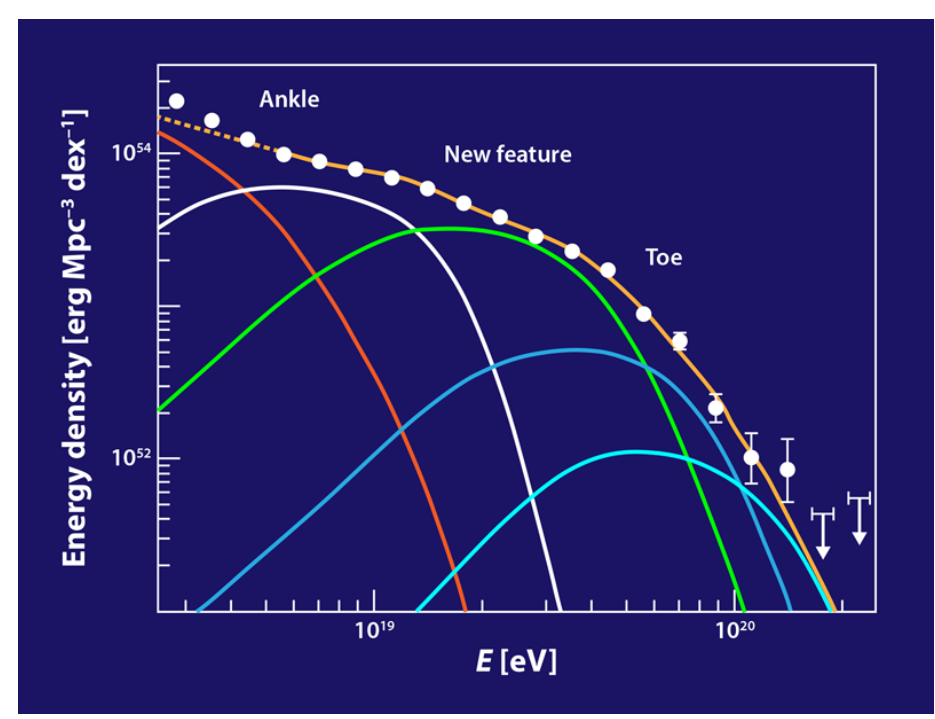

Figure 2: The UHECR spectrum compiled from the latest Auger data is shown here in terms of the energy density. The observations reveal a new feature at $13 \times 10^{18} \mathrm{eV}$, where the spectrum steepens slightly. This break in the power-law fit occurs between two other breaks: an ankle and a toe. The shape of the spectrum suggests that different nuclei (shown in colors) contribute at different energies.

Credit: APS/Alan Stonebraker; adapted from Ref. [2].

heavier masses like that of iron. At higher energies, ground-based arrays must use computer simulations to decipher which primary particle likely produced an EAS. For example, previous work using Auger data suggested that protons dominate the cosmic-ray composition at energies of $5 \times 10^{18} \mathrm{eV}$, the location of the so-called ankle where the UHECR spectrum flattens out briefly. Toward higher energies, the simulations infer that the composition becomes progressively heavier, going through helium, carbon, nitrogen, oxygen, silicon, and (perhaps) iron nuclei. However, these sorts of simulations rely on models of hadronic (quark-containing) interactions, which are only verified up to $10^{17}$-eV lab energy using LHC measurements. Extrapolations to higher energies are uncertain, and small changes in the models can result in a "proton-only" interpretation of the shower data [5].

Furthermore, observations from the Auger and TA are not fully compatible with each other, as simulations based on TA data prefer a predominantly light composition consisting of mostly protons and helium nuclei [6].
The latest Auger data could help clear up the issue [2]. The collaboration has compiled all their events above $2.5 \times 10^{18} \mathrm{eV}$ into a spectrum, which they find is better characterized by a four-component power-law fit than the previously used three-component fit [7]. The new feature is a steepening-or softening-of the spectrum at an energy of $13 \times 10^{18} \mathrm{eV}$. This break occurs between the ankle at $5 \times 10^{18} \mathrm{eV}$ and the toe at $46 \times 10^{18} \mathrm{eV}$, where the spectrum steepens very sharply (Fig. 2). The softening of the spectrum in this intermediate region could be a hint that the mass composition of UHECRs is changing from light to heavy. Such an interpretation assumes that the UHECR spectrum is dominated by different elements at different energies. This type of model requires that the UHECR sources accelerate particles with an extremely hard (or flat) spectrum [8], and that the number of these sources either remains the same over cosmic time or was even fewer at earlier times [9]. However, both of these requirements are at odds with our knowledge of luminous astrophysical sources from observations in radio to gamma rays.

A more profound result from the latest Auger data is the near independence of the spectral shape on the angle with respect to the celestial equator [2]. This lack of anisotropy in the arrival directions severely disfavors models that assume that all the UHECRs are produced by a few bright and nearby sources. Instead, the data favor a uniform spatial distribution of UHECR sources, which implies they are extragalactic. To account for the observed UHECR flux, these sources must be injecting energy into the Universe at a rate of approximately $6 \times 10^{44} \mathrm{erg} \mathrm{Mpc}^{-3} \mathrm{yr}^{-1}$. Observations in gamma rays with energies above $100 \mathrm{MeV}$ suggest only a few source classes that can supply that much energy, namely, radio galaxies of Fanaroff-Riley type-I, BL Lac objects, and starburst galaxies [10]. The first two are high-energy subclasses of active galactic nuclei (AGN) that have strong jets for possibly accelerating particles. But if indeed the UHECRs are rich in heavy nuclei, then this poses a challenge for AGN scenarios. First, it is not clear how an AGN can produce heavy nuclei in its jet, and second, it is hard to see how those nuclei might be accelerated without breaking up in interactions with the radiation field that surrounds the AGN. By contrast, it is relatively easy to find an environment within starburst galaxies that is rich in heavy elements and has bursting sources that can accelerate these nuclei to ultrahigh energies [11]. 
To establish this overall picture of starburst galaxies as sources, we will need further evidence that heavy nuclei make up the dominant fraction of the UHECRs above the ankle. Such confirmation will require further observations of the sky and also a better understanding of particle physics interactions seen in collider experiments. The field of UHECRs has now entered into an era of precision measurements that are challenging our knowledge in both physics and astrophysics.

Correction (2 November 2020): An earlier version stated that models of hadronic interactions were verified up to $10^{17}-\mathrm{eV}$ center-of-mass energy, when in fact this value corresponds to the lab energy.

Soebur Razzaque: Centre for Astro-Particle Physics (CAPP) and Department of Physics, University of Johannesburg, Johannesburg, South Africa

\section{REFERENCES}

1. J. Linsley et al., "Extremely energetic cosmic-ray event," Phys. Rev. Lett. 6, 485 (1961).

2. A. Aab et al. (Pierre Auger Collaboration), "Features of the energy spectrum of cosmic rays above $2.5 \times 10^{18} \mathrm{eV}$ using the Pierre Auger Observatory," Phys. Rev. Lett. 125, 121106 (2020).

3. A. Aab et al. (Pierre Auger Collaboration), "The Pierre Auger
Cosmic Ray Observatory," Nucl. Instrum. Methods A 798, 172 (2015).

4. T. Abu-Zayyad et al. (Telescope Array Collaboration), "The surface detector array of the Telescope Array experiment," Nucl. Instrum. Methods A 689, 87 (2012).

5. G. R. Farrar, "Particle physics at ultrahigh energies," arXiv:1902.11271

6. W. Hanlon (Telescope Array Collaboration), "Telescope array 10 year composition,” Proc. Sci. ICRC2019, 280 (2020).

7. A. Aab et al. (Pierre Auger Collaboration), "Measurement of the cosmic-ray energy spectrum above $2.5 \times 10^{18} \mathrm{eV}$ using the Pierre Auger Observatory," Phys. Rev. D 102, 062005 (2020).

8. R. Aloisio et al., "Ultra high energy cosmic rays: Implications of Auger data for source spectra and chemical composition," J. Cosmol. Astropart. Phys. 10, 020 (2014).

9. A. M. Taylor et al., "Indications of negative evolution for the sources of the highest energy cosmic rays," Phys. Rev. D 92, 063011 (2015).

10. C. D. Dermer and S. Razzaque, "Acceleration of ultra-high-energy cosmic rays in the colliding shells of blazars and gamma-ray bursts: Constraints from the Fermi Gamma-ray Space Telescope,” Astrophys. J. 724, 1366 (2010). 11. X.-Y. Wang et al., "On the origin and survival of ultra-high-energy cosmic-ray nuclei in gamma-ray bursts and hypernovae," Astrophys. J. 677, 432 (2008). 